\title{
Integración y Escuela: Hacia la Diversidad
}

\section{Integration and School: Towards Diversity}

\author{
Lourdes Ilizástigui del Portal ${ }^{1}$ \\ Universidad Santo Tomás de Chile
}

(Recepción: Septiembre 2005 - Aceptación: Noviembre 2005)

\begin{abstract}
Una revisión de los rasgos conceptuales del fenómeno de Integración de los niños discapacitados revela que éste supone un proceso más complejo de lo que podemos imaginar. Se pone en tela de juicio el sistema educativo tradicional y se aspira a una educación nueva y renovada, flexible, que responda a las necesidades educativas especiales de todos los escolares. Como proceso, transita por un camino largo, por etapas de cambios graduales y prácticas en que el actual sistema educativo asimila la capacidad integradora hacia el ideal de la escuela abierta a la diversidad. ¿Cómo lograr la integración?, ¿qué métodos implementar?, ¿qué prácticas educativas seguir? Son preguntas que aún no están respondidas totalmente y que los profesionales vinculados a este ámbito nos hacemos cada día.
\end{abstract}

Palabras clave: Integración, discapacidad, diversidad.

\begin{abstract}
A review of the conceptual features of integrating handicapped children reveals that it is a more complex process than we can imagine. It assesses the traditional educational system and aspires to a new, renovated system, one that is flexible and responds to the special educational needs of all school children. As a process, it is a long road that entails gradual changes and practices in which the current educational system moves slowly toward an integrative capacity that is the ideal for a school system open to diversity. How is this integration to be achieved? What methods should be used? What educational practices should be followed? These are questions that have not yet been fully answered and which we educational professionals ask ourselves every day.
\end{abstract}

Key words: Integration, handicap, diversity

\section{Introducción}

Ante la posibilidad de ofrecer algunas reflexiones en torno a la Integración y la escuela, deseo manifestar que soy consciente de los inconvenientes y ventajas que pueden representar, ya que en la escuela regular y la escuela especial son evidentes en la actualidad las discrepancias y no coincidencias de los profesionales respecto a la respuesta educativa de los niños discapacitados. No obstante, en esta ocasión pretendemos intercambiar nuestra visión en torno al tema, aprovechando la oportunidad de contrastar constructivamente ideas con profesionales que día a día se enfrentan a esta hermosa y humana actividad.

Considero que resulta relevante abordar el problema de la integración y su complejidad, la educación y desarrollo de la personalidad del discapacitado en escuela regular así como la Diversidad, que, a mi juicio, configuran ejes esenciales que determinan una concepción teórica metodológica y práctica para guiar y orien-

1 Psicóloga, Licenciada en Psicología, Universidad de La Habana. Magister en Educación Especial, Universidad de La Habana-Centro de Referencia Latinoamericano de la Educación Especial. E-mail: lilizastigui@santotomas.cl tar la respuesta educativa a los niños con necesidades educativas, discapacitados o no.

Una revisión de los rasgos conceptuales del fenómeno de la Integración de los niños discapacitados revela que supone un proceso más complejo de lo que podemos imaginar. Se pone en tela de juicio el sistema educativo tradicional y se aspira a una educación nueva y renovada, flexible, que responda a las necesidades educativas especiales de todos los escolares. Resulta un proyecto complejo pero no irrealizable como se ha demostrado. Como proceso, transita por un camino largo, por etapas de cambios graduales y prácticas en que el actual sistema educativo asimila la capacidad integradora hacia el ideal de la escuela abierta a la diversidad.

¿Cómo lograr la integración?, ¿qué proceso de evaluación seguir?, ¿qué métodos implementar? ¿Qué prácticas educativas seguir? Son preguntas que aún no están respondidas totalmente y que los profesionales vinculados a este ámbito nos hacemos cada día.

Me parece que tenemos clara la idea reguladora de la Integración y en la práctica algunas modalidades de respuesta educativa, pero es imprescindible que configuremos una concepción teórica metodológica más flexible, optimis- 
ta y ajustada a las necesidades y recursos propios del medio donde se desarrollan.

Nuestro principal mensaje:

- Reformular concepciones y actitudes acerca del desarrollo y aprendizaje del niño discapacitado.

- Inmovilidad versus movilidad institucional.

- Flexibilidad ante los desafíos de hoy.

- Intencionar en nuestras escuelas más preguntas que respuestas frente al desarrollo y aprendizaje de los escolares.

- Configurar la personalidad del niño discapacitado en una educación que se sustente en el potencial y no en el déficit.

- La Interdisciplinariedad como vía para el desarrollo potencial.

\section{El Problema y su Complejidad}

La caracterización del niño con necesidades educativas especiales es de complejidad singular y afecta la evaluación, el diagnóstico y la intervención. Estos niños constituyen una proporción importante de la población escolar. En ellos se generan problemas, limitaciones, sentimientos de minusvalía que resultan penosos, los hacen sufrir a ellos y a sus familiares, exigen recursos adicionales, materiales y humanos de los sistemas de educación, que están obligados a prestar servicios especiales que garanticen el desarrollo de las capacidades potenciales de estos niños y faciliten su inserción social en la vida laboral activa.

La complejidad del dominio que nos ocupa está determinada por razones de distinta índole:

- Clasificar y etiquetar a un niño con necesidades educativas especiales contribuyen a su marginación, y la clasificación según etiología se enmarca en un modelo médico tradicional.

Este modelo considera al discapacitado como portador de deficiencias, como un enfermo, y no tiene en cuenta (según críticas del movimiento integracionista) causas de orden cultural. Se critica de este modelo el hecho de que se centra en las deficiencias, en los defectos y que su traducción en la práctica (como diagnóstico) es muy costosa.

- Conocer los déficit y la etiología no implica necesariamente hacer énfasis sólo en ellos y no en las potencialidades. Estas últimas es- tán estrechamente interrelacionadas con los propios defectos. Ambos constituyen dos caras de una misma moneda.

- Los instrumentos, tests que en la actualidad se emplean en el proceso de evaluación no dan cuenta del potencial del niño, ya que se sustentan en un referente de capacidades estáticas, por lo que es necesario incluir metódicas cualitativas que permiten conocer la ZONA DE DESARROLLO PRÓXIMO.

- El tratamiento de los niños discapacitados atañe no a la multidisplinariedad, sino a la interdisciplinariedad, que requiere el diseño de un sistema de influencias coherente y sistemático con el compromiso de todos los agentes educativos incluyendo la movilidad institucional en su rol de mediador para facilitar el desarrollo y aprendizaje de estos niños.

- El proceso de evaluación y diagnóstico necesita integrar los aportes de de la Evaluación Dinámica y Participativa.

Las particularidades de la exploración clínico-psicológica infantil imponen un sujeto activo, móvil, cambiante en evolución que está en interrelación y que hace indispensable el pleno conocimiento y aplicación consecuente de los aportes teóricos y actuales acerca del desarrollo y aprendizaje.

Durante los últimos años ha aumentado el interés de los profesionales por perfeccionar el trabajo de Evaluación, Diagnóstico e Intervención. Alcanzar este propósito requiere no sólo garantizar los métodos, técnicas y detección temprana que permitan una evaluación más precisa, sino demanda una concepción teórica-metodológica contextualizada, participativa y op timista en el contexto escolar.

\section{Evaluación Participativa en el Contexto Escolar}

- Rol activo del niño: el niño no sólo construye, también re-construye en la medida en que se involucra en su autotransformación en el contexto de una interrelación de calidad. Es decir, donde se le intencione, se le signifique y pueda trascender así lo aprendido, a otras esferas de la vida.

- El aprendizaje como guía del desarrollo: el aprendizaje impulsa el tránsito de un nivel de desarrollo a otro. 
- Zona de Desarrollo Próximo: Significa no sólo el avance hacia una nueva forma de considerar la relación entre enseñanza y aprendizaje sino que constituye un instrumento metodológico de concebir la evaluación y diagnóstico del desarrollo como proceso formativo y no únicamente como estimación de productos.

- Mediación: no sólo como el mecanismo o proceso que produce el desarrollo, sino que el énfasis debe estar en la apropiación de la mediación por el sujeto, es decir, que tenga lugar un proceso formativo del plano interno y de la función propiamente dicha de la subjetividad.

- La Interdisciplinariedad: potenciador en el conocimiento y transformación de la subjetividad e intersubjetividad en un contexto determinado.

La Evaluación Participativa es una metodología contextualizada, participativa, potenciadora y continua en el contexto escolar.

En la alternativa que proponemos, déficit y potencialidades constituyen dos aspectos de un mismo problema.

La comprensión de la capacidad potencial de aprendizaje del niño discapacitado puede ser evaluada desde las Metódicas Cualitativas. Durante el proceso de evaluación, el mediador constata la capacidad del menor para resolver la actividad de modo independiente; si no lo logra, proporciona niveles de ayuda que el niño debe asimilar y transferir a una nueva situación, lo que en este caso resulta esencial-dando cuenta del potencial que posee.

Educación y Desarrollo de la Personalidad del Discapacitado en la Escuela Regular.

La personalidad en su concepción más general se considera un sistema integro y jerárquico que determina una conducta dada, que permite no sólo la adaptación, construcción y reconstrucción de la realidad sino la trasformación y autotrasformación del sujeto.

Todo este proceso ocurre gracias a la interrelación de los aspectos biológicos y sociales y en su centro se ubica la educación familiar y escolar.

Tanto la actividad como la comunicación tienen un inmenso significado en la psicología de la personalidad, ya que posibilitan la formación de los procesos y cualidades psíquicas del hombre.

De manera que la personalidad se considera un producto tardío del desarrollo psíquico, pues alcanza cierto nivel de madurez en la juventud y la adultez; no obstante, todos sus componentes se comienzan a formar desde muy temprano.

Las investigaciones de diversos autores han demostrado que el desarrollo de la psiquis de los niños discapacitados transcurre de acuerdo con los patrones y regularidades más generales del desarrollo psíquico, aunque este proceso adquiere una expresión peculiar, demuestra tener rasgos característicos y presenta múltiples insuficiencias, debido a la existencia de un conjunto de factores atípicos. La complejidad de la estructura y del desarrollo de la psiquis del niño discapacitado está dada porque los defectos secundarios que surgen a consecuencia del defecto primario, provocan cambios en todo el proceso de formación de la personalidad, sin llegar a violar las leyes generales del desarrollo psíquico.

La personalidad del niño discapacitado se va formando, al igual que la de los niños sin alteraciones en su desarrollo, bajo las mismas leyes, las cuales actúan a través de sus condiciones internas, que constituyen premisas indispensables y cuyas consecuencias en el orden cultural intensifican, nutren y fijan el propio defecto; no sólo cambia la actitud del hombre hacia el mundo sino que también, y ante todo, influyen en las relaciones con las personas.

Partiendo de que en este contexto se desarrolla una personalidad matizada por características peculiares, de hecho se considera que integralmente el discapacitado puede lograr determinado nivel de desarrollo de su personalidad y de cualidades positivas y suficientemente estables, mediante vías, métodos y procedimientos especiales del trabajo educativo. Dentro de este contexto resulta de gran valor la influencia pedagógica en el conjunto de las influencias sociales. La escuela regular se constituye como medio natural para formarlos integralmente como individuos y atenuar al máximo las desviaciones en el desarrollo de la personalidad.

En este contexto, la relación de la escuela con los padres como agentes educativos esenciales para el desarrollo de la personalidad adquiere una particular importancia, lo cual se explica por lo siguiente:

1. Los propios padres son muchas veces portadores de creencias y prejuicios dañinos para la autovaloración del escolar discapacitado.

2. De acuerdo a la propia necesidad educativa, con frecuencia el escolar necesita un apoyo 
extra en la realización de sus tareas, así como un estímulo y una atmósfera positiva en su hogar para su desempeño en las mismas, lo que muchas veces no es asumido por los padres.

3. El diálogo con los padres, conservado a través del tiempo por la habilidad que ellos tengan para respetar y seguir el proceso de necesidades cambiantes de los hijos, es un elemento importante para el bienestar y seguridad emocional de los escolares. Sin embargo, con mucha frecuencia el diálogo está totalmente ausente en la relación padre e hijo.

El trabajo con los padres dirigido a implicarlos en una buena educación del niño con discapacidad no se puede reducir a la información sobre el hijo como se ha hecho tradicionalmente. Es preciso intencionar, la reflexión acerca de cuánto más se puede hacer por el desarrollo.

El diálogo con los padres debe ser natural, donde ellos no sientan que queremos enseñarles lo que deben hacer, sino nuestro esfuerzo por el desarrollo del menor y que representa una vía fundamental para el cambio de creencias erróneas y estereotipos de los padres, que conduce a nuevos niveles en la relación con el escolar.

El desarrollo de la personalidad en el discapacitado es un factor esencial para su avance en el aprendizaje que, de no lograrse de forma simultánea con el desarrollo del conocimiento, puede impedir la conversión de éste en un sujeto con habilidades y capacidades estables que garanticen el avance del discapacitado y su integración plena.

\section{Principios para la Educación de la Personalidad del Discapacitado}

1. De la Personalidad: centrar el proceso educativo en la personalidad de los estudiantes y en su carácter activo, al considerar este como sujeto de la actividad pedagógica, con todas sus características personales concretas e irrepetibles.

2. De la unidad de lo cognitivo y afectivo: es imposible delimitar un hecho o fenómeno del mundo fragmentado. Por esto las actividades de conocimientos de los estudiantes tienen que ser portadoras de un contenido emocional favorable para poder cumplir los objetivos educativos.

3. De la unidad de la actividad y la comunicación: constituyen reguladores de la perso- nalidad. Una de las condiciones para el éxito de la labor pedagógica radica en la calidad de las actividades que realizan los estudiantes, junto a sus profesores y la fluida comunicación que establezcan ambos como también los estudiantes entre sí.

4. De la unidad de las influencias educativas: el proceso educativo no es privativo de la escuela, aunque ella ocupe el papel rector en el mismo. En dicho proceso intervienen otros, por lo que debe existir una relación colaborativa para que las influencias se correspondan con las funciones de cada uno.

5. De la unidad de lo instructivo y lo educativo: lo instructivo debe repercutir en la personalidad, de acuerdo con los objetivos de la educación, pero si se es consciente de esa unidad y se es consecuente con ella, en todo proceder del profesor y de la escuela debe existir unidad entre ambas, de lo contrario quedan los conocimientos, valores y habilidades como algo externo al complejo mundo motivacional, lo que no estimula el desarrollo de la personalidad ni en la formación de convicciones que regulen su conducta.

6. Del carácter grupal e individual de la educación: en el proceso grupal se forma y se transforma el desarrollo de la personalidad de manera que la actividad educativa deberá realizar actividades tanto individuales grupales.

7. De la vinculación de la educación con la vida: las materias no constituyen un fin en sí mismas, sino un medio para lograr la inserción social, por lo que debe constituirse en una estrategia educativa.

8. De la incorporación y participación activa del niño en el proceso de educación y reeducación. Se trata no sólo de incluir al niño en el aprendizaje sino en el proceso de su educación.

Algunas recomendaciones prácticas para la educación de la personalidad: respuesta educativa.

- Conocer primero la personalidad de los estudiantes y la manera de actuar antes de plantearse influir sobre ella. Partir siempre de una caracterización integral.

- Estimular la aparición y el desarrollo de diferentes cualidades de la personalidad en sus relaciones y condicionamiento mutuos.

- Desarrollar una orientación activa y trasformadora en los profesores. 
- Respetar la personalidad de los estudiantes.

- Planificar el trabajo educativo con el fin de incrementar la autorregulación de la personalidad.

- Crear en las actividades docentes extraprogramáticas un clima socio-psicológico favorable y participativo con los estudiantes, proclive al aprendizaje y a la interacción.

- Ser capaz el profesor de diagnosticar de forma acertada el nivel motivacional de los estudiantes para las actividades del proceso de enseñanza-aprendizaje.

- Constatar cómo los conocimientos trasmitidos a los estudiantes se van personalizando progresivamente e influyen en el desarrollo de la regulación y autorregulación de su conducta.

- Todas las actividades docentes deben despertar emociones y sentimientos positivos en los estudiantes.

- Tener en cuenta los gustos, intereses, motivos y necesidades de los estudiantes al planificar diferentes actividades.

- Favorecer y estimular los éxitos individuales y grupales de los estudiantes.

- Incentivar a los educandos para que apliquen sus conocimientos en la solución de problemas de diferente naturaleza y en diferentes ámbitos.

- Planificar y ejecutar actividades docentes, extraprogramáticas que propicien la comunicación interpersonal entre los estudiantes y con los profesores.

- Diagnosticar las posibles barreras de la comunicación.

- No prejuiciarse con ningún estudiante.

- Orientar con un lenguaje claro y preciso la actividad concreta por ejecutar.

- Precisar qué tipo de acciones incluye la actividad.

- Aclarar con qué medios los estudiantes cuentan para desarrollar la actividad.

- Analizar con los estudiantes las actividades desarrolladoras, destacando los éxitos y dificultades individuales y grupales.

Es evidente la estrecha relación interna que existe entre todos los principios, pues el cumplimiento de uno favorece la aplicación de otros; por el contrario, la violación de alguno influye en el incumplimiento de otro. En la realidad educativa todos giran alrededor del desarrollo de la personalidad del estudiante y se concentran integralmente en él, de ahí su interdependencia y unidad.

\section{Tenemos que Enseñar a Vivir en la Diversidad}

En los últimos tiempos muchos profesionales han centrado sus esfuerzos en la atención de la diversidad, apoyo a la diversidad, a favor de la diversidad, educación en y para la diversidad; pero este hecho no implica que en nuestras escuelas esté insertada la diversidad.

La diversidad debe configurarse como una de las dimensiones centrales en los cambios que la educación actual debe enfrentar. ¿Y cuáles son las razones?

Las principales razones son que la diversidad es una realidad social ineludible, que la educación no puede quedar descontextualizada de la pluriculturalidad. La construcción de una sociedad democrática en su pleno sentido impone la diversidad como esencia de la educación y la diversidad entendida como un valor positivo presupone un reto para el enriquecimiento de los procesos educativos en general y de las posibilidades didáctico-metodológicas en particular.

Indiscutiblemente la postura Integracionista ha dejado atrás la segregación y un enfoque centrado en el déficit, pero aún hay numerosas reticencias a hacer un cambio de fondo que de verdad cambie las concepciones y actitudes de los distintos sectores implicados en la educación.

La diversidad es un factor inherente a la naturaleza humana, positivo, valioso, enriquecedor y fuente de progreso educativo. Una postura educativa que se sitúa más allá de la integración no sólo da respuesta educativa a los estudiantes con necesidades educativas especiales, discapacitados o no en la escuela regular, sino que enseña a vivir y no establecería distinciones entre los estudiantes, sería innecesario.

En mi opinión existen condiciones que favorecen la Integración como tránsito hacia una escuela comprensiva y diversa:

- Un liderazgo efectivo, no solamente del director sino extendido a lo largo de toda la escuela.

- Participación del profesorado, estudiantes y comunidad en las políticas y decisiones de la escuela. 
- Un compromiso para una planificación colaborativa.

- Estrategias de coordinación.

- Atención a los benefícios potenciales de la pregunta y la reflexión.

- Una plan de desarrollo del profesorado.

"La función educativa de la escuela requiere una comunidad de vida, de participación democrática, de búsqueda intelectual, de diálogo y aprendizaje compartido, de discusión abierta sobre la bondad y sentido antropológico de los influjos del proceso de socialización. Una comunidad educativa que rompa las absurdas barreras artificiales entre la escuela y la sociedad. Un centro educativo flexible y abierto donde colaboran los miembros más activos de la comunidad para recrear la cultura, donde se apren- de porque se vive, porque vivir democráticamente significa participar, construir cooperativamente alternativas a los problemas sociales e individuales, fomentar la iniciativa, integrar diferentes propuestas y tolerar las discrepancias" (Pérez Gómez, 1997: 52).

\section{Referencias}

ILIZÁSTIGUI, L. (2002) La integración Social del Discapacitado ¿Teoría o Realidad? Art. no publicado.

JIMÉNEZ, P., VILA, M. (1999) De Educación Especial a la Educación en la Diversidad. Ed. Aljibe.

MINEDUC (1999) Integración Escolar de alumnos y alumnas con necesidades educativas especiales. Reglamento de Educación. Ley de Integración Social de personas discapacitadas.

VIGOTSKY, L. S. (2002) El desarrollo de los procesos psicológicos superiores. Barcelona: Editorial Crítica. 